\title{
Mortalidad de vertebrados en la carretera Guanare-Guanarito, estado Portuguesa, Venezuela
}

\author{
Andrés Eloy Seijas ${ }^{1}$, Alexis Araujo-Quintero ${ }^{2} \&$ Nadines Velásquez ${ }^{1}$ \\ 1. Universidad Nacional Experimental de los Llanos Occidentales "Ezequiel Zamora" (UNELLEZ), Antiguo Convento \\ de San Francisco, Carrera 3, Guanare, Portuguesa; aeseijas@gmail.com, nadines06@gmail.com \\ 2. Museo de Ciencias Naturales de Guanare (MCNG-UNELLEZ), Mesa de Cavacas, Guanare, Portuguesa; \\ arome101@yahoo.com
}

Recibido 19-X-2012. Corregido 20-II-2013. Aceptado 13-III-2013.

\begin{abstract}
Vertebrate mortality in the Guanare-Guanarito road, Portuguesa state, Venezuela. Roads directly or indirectly affect the structure, dynamics and function of ecosystems that they traverse. Most studies on the effect of roads on wildlife focus on the evaluation of mortality of vertebrates by vehicle collisions. Despite the extensive road network that exists in Venezuela, studies of wildlife mortality in them are scarce. In this paper, we analyzed the temporal and spatial pattern of vertebrate's collisions along the road Guanare-Guanarito, in Portuguesa state. We travelled 26 times between these towns $(74 \mathrm{~km})$ to localize dead vertebrates, at a speed of $50-60 \mathrm{~km} / \mathrm{h}$. of those trips were conducted from March 13 to October 26, 2010, and 10 additional trips from December 7, 2009 to December 14, 2010; these ones, with the aim to include months and seasons that were insufficiently sampled during the first period. The elapsed time between trips varied from 14 to 37 days. The total distance traveled was $1924 \mathrm{~km}$. Dead animals found amounted 464 individuals, 66 of them were birds (25 identified species), 130 mammals ( 15 species) and 268 reptiles (18 species). The species with the highest number of individuals were the snake Leptodeira annulata $(\mathrm{n}=119)$, the oppossum Didelphis marsupialis $(\mathrm{n}=39)$ and the spectacled caiman Caiman crocodilus $(\mathrm{n}=33)$. Excluding domestic animals, the rate of road-killed vertebrates was $0.2282 \mathrm{indiv} . / \mathrm{km}$, a figure $28.3 \%$ higher than previous studies in the same road. Changes in the relative number of collisions for some species, respect to the numbers reported 20 years ago, were linked to the increase in traffic flow and changes in land use. Road segments with collision rates higher than expected by chance were identified. Collition by cars may be the principal cause of mortality for species like the tamandua (Tamandua tetradactyla) and the giant anteater (Myrmecophaga tridactyla), the last considered a vulnerable species. Some basic measures are proposed to reduce wildlife mortality on the road. Rev. Biol. Trop. 61 (4): 1619-1636. Epub 2013 December 01.
\end{abstract}

Key words: roadkills, wildlife conservation, Venezuelan Llanos, Leptodeira annulata, Myrmecophaga tridactyla.

Las carreteras y autopistas causan un impacto en los hábitats y ecosistemas que no se circunscribe exclusivamente al espacio directamente afectado en el momento que son construidas. Estas infraestructuras afectan directa o indirectamente la estructura, dinámica, función y composición de especies de los ecosistemas que atraviesan (Spellerberg, 1998; Carr, Fahrig \& Pope, 2002; Clevenger, Chruszcz, \& Gunson, 2003; Coffin, 2007). Entre los impactos generados o inducidos por las carreteras tenemos la destrucción de flora y fauna al momento de la construcción, la modificación y alteración del ambiente físico y químico en su entorno inmediato, la modificación del comportamiento de las especies, la facilitación del proceso de dispersión de especies autóctonas y exóticas, la aceleración de cambios en el uso de la tierra por los seres humanos y la pérdida de conectividad entre ecosistemas por la fragmentación 
y el efecto barrera (Forman \& Alexander, 1998; Trombulak \& Frissell, 2000; Arévalo \& Newhard, 2011).

Uno de los aspectos más investigados en relación a la "ecología de la carretera" es el atropellamiento de vertebrados silvestres (Coffin, 2007). Esos estudios han sido realizados principalmente en Norteamérica y Europa (Madsen, Strandgaard \& Prang, 2002; Pokorny, 2006; Grilo, Bissonette \& Santos-Reis, 2009), aunque en los últimos años se ha incrementado el interés sobre este tema en los países del Neotrópico, particularmente en Brasil (Delgado, 2007; Coelho, Kindel, \& Coelho, 2008; Turci \& Bernarde, 2009; Gumier-Costa \& Sperper, 2009; Bager \& Rosa, 2010; Vargas-Salinas, Delgado-Ospina \& López-Aranda, 2011; Quintero-Ángel et al., 2012). En el caso de Venezuela sólo se conocen tres trabajos publicados en revistas arbitradas (Ramo \& Busto, 1986; Rodda, 1989; Pinowski, 2005).

Debido al conocido impacto de las carreteras sobre los ecosistemas en general y sobre la fauna silvestre en particular, se hace necesario implementar medidas de mitigación. Sin embargo, esas medidas sólo pueden ser llevadas a cabo de manera efectiva cuando están basadas en estudios detallados que determinen, entre otras cosas: la periodicidad de los atropellos, su relación con el flujo vehicular, las especies más afectadas, la disposición espacial de los atropellos y su relación con las características del paisaje a lo largo del cual la carretera transcurre (Clevenger et al., 2003; Andrews \& Gibbons, 2005).

Por otra parte, el análisis de datos sobre animales atropellados en las carreteras puede servir para determinar tendencias al largo plazo de poblaciones de algunas especies o incluso como sustituto o complemento de otros métodos de evaluación (Case, 1978; Gehrt, Huber \& Ellis, 2002). En este artículo se analiza la mortalidad de fauna por atropellamiento en una carretera de los Llanos Occidentales de Venezuela en cuanto a frecuencia por especies, patrón temporal y características del hábitat en los lugares de atropello. Los resultados se comparan con los de investigaciones previas llevadas a cabo en la misma vía en los años 1980-1982 (Ramo \& Busto, 1986) y 1991-1992 (Pacheco, 1993) con el fin de determinar si han ocurrido cambios en la importancia relativa de las especies afectadas.

\section{MATERIALES Y MÉTODOS}

Área de estudio: La carretera estudiada se localiza en el estado Portuguesa, Venezuela, y une en sus extremos a las capitales de los municipios Guanare (157470 habitantes) y Guanarito (25 109 habitantes), pero la mayor parte de su trayectoria (72\%) transcurre a través del Municipio Papelón (13090 habitantes) (Fundación para el Desarrollo de la Región Centro Occidental [FUDECO], 2004). La vía, construida en 1972, tiene aproximadamente $8 \mathrm{~m}$ de ancho y $80 \mathrm{~km}$ de longitud. La carretera es por lo general recta con algunos sectores de curvas moderadas. Por ella circulan todo tipo de vehículos, con predominancia de automóviles particulares y camiones que transportan mercancías y productos propios de la región. El límite de velocidad establecido es de $80 \mathrm{~km} / \mathrm{h}$, pero no hay vigilancia que obligue a su cumplimiento.

No existen datos oficiales actualizados sobre el flujo de vehículos en la carretera Guanare-Guanarito, pero asumimos que éste ha incrementado de manera progresiva a través de los años, lo cual se corresponde con una tendencia mundial (Carr et al. 2002). No obstante, a partir de datos en Ramo \& Busto (1986) para el lapso 1972-1980 se desarrolló el modelo: $y=108.78 x-213684\left(R^{2}=0.98\right)$, donde " $y$ " es el número de vehículos y " $\mathrm{x}$ " es el año. Con esta ecuación se puede calcular que el incremento en el flujo de vehículos en el lapso transcurrido desde el estudio de Pacheco (1993) (años 19911992) y el presente estudio (años 2008-2010) fue de $64.5 \%$.

En el paisaje que bordea la Carretera Guanare-Guanarito predomina una matriz de pastizales y sabanas, con relictos boscosos, cultivos y otros tipos de cobertura. En el momento en que Ramo \& Busto (1986) llevaron a cabo su estudio sobre la fauna atropellada (19801982), esta vía atravesaba "una zona boscosa, 
aclarada con numerosos campos de cultivo" (p. 33). Pacheco (1993) también señaló que a comienzos de los años noventa del siglo pasado, los bosques constituían el tipo de cobertura predominante, pero que parte de ellos ya estaban intervenidos y alterados. Aunque no existen investigaciones locales que lo cuantifiquen, se puede presumir que en los últimos cincuenta años, la explotación de madera, la ganadería extensiva y la actividad agrícola ha reducido y fragmentado el bosque original, como ha ocurrido en todos los Llanos de Venezuela (Aymard, Farreras \& Schargel, 2011; PachecoAngulo, Aguado-Suárez \& Mollicone, 2011).

La principal actividad económica de la región es la ganadería extensiva, seguida por la agricultura. El régimen de precipitación presenta una temporada seca, desde diciembre hasta marzo y otra lluviosa, entre mayo y octubre, con una precipitación media anual de $1375 \mathrm{~mm}$ (Solórzano et al., 2006). Noviembre y abril son de transición entre las mencionadas temporadas. El relieve es plano e irregular, con una pendiente norte-sur menor a $1 \%$, y una altura sobre el nivel del mar de $80 \mathrm{~m}$. En la época lluviosa se inundan extensas áreas de las zonas más bajas del terreno. A las orillas de la vía se observan pequeñas lagunas y "préstamos", que son cuerpos de agua formados en las excavaciones de donde se extrajo tierra para elevar el terreno donde se construyó la carretera. Dichos préstamos conservan agua durante la época seca.

Muestreos: Los recorridos (o muestreos) para la localización de reptiles, aves y mamíferos atropellados se iniciaron en la mañana (entre las 05:43 y 06:31hr) debajo del puente de la autopista José Antonio Páez en Guanare (901'24.1' N - 69043'55.7' W) y finalizaron en el puente sobre el río Guanare $\left(8^{\circ} 42^{\prime} 30.2^{\prime \prime}\right.$ $\left.69^{\circ} 12^{\prime} 45.4^{\prime \prime} \mathrm{W}\right)$ en la población de Guanarito (entre las 08:15 y las 09:00hr) cubriendo una distancia de $74 \mathrm{~km}$. La hora de inicio varió de acuerdo con la época del año y las condiciones de visibilidad al amanecer para el día del muestreo; la de hora de finalización dependió esencialmente del número de animales localizados en cada recorrido. Se viajó a una velocidad de $50-60 \mathrm{~km} / \mathrm{h}$.

Se efectuaron 16 recorridos en el lapso que va desde el 13 de marzo hasta el 26 de octubre 2008, y 10 adicionales desde el 7 de diciembre 2009 hasta el 14 de diciembre 2010; éstos últimos llevados a cabo para completar meses y periodos climáticos insuficientemente cubiertos durante el primer lapso. El tiempo transcurrido entre muestreos varió entre 14 y 21 días en el primer lapso y 14 y 37 días para el segundo. El número total de kilómetros transitados (una sola dirección) fue de $1924 \mathrm{~km}$ y se llevaron a cabo $54 \mathrm{hr}$ de observación.

Para cada animal encontrado sobre el asfalto se anotó: especie, hora y fecha de observación. Su localización se determinó con un GPS Garmin 12, en coordenadas UTM Datum WGS84. Se tomaron fotografías que facilitaron la identificación de los individuos. La ubicación con el GPS y las fotografías se usaron para evitar el conteo repetido de los especímenes localizados en muestreos sucesivos. Aquellos que por su mal estado no pudieron ser identificados in situ, fueron trasladados para compararlos con los especímenes depositados en la colección del Museo de Ciencias Naturales de Guanare (MCNG) y luego fueron descartados.

La tasa de atropellos (TA) se calculó dividiendo el número total de cadáveres encontrados entre el número de $\mathrm{km}$ recorridos (Frías, 1999; Bager \& Rosa, 2010; Glista \& DeVault, 2008; Cunha, Moreira \& Silva, 2010; Hegel, Consalter \& Zanella, 2012). Asumiendo que los animales fueron atropellados entre las 24 horas previas a su localización, estas tasas son equivalentes a tasas de mortalidad diaria.

Se elaboraron curvas de rarefacción para cada uno de los grupos vertebrados estudiados. Estas curvas representan el incremento en el número de especies al aumentar el esfuerzo de muestreo y, en este caso, informan sobre la medida en que las especies presentes en el área de estudio están representadas entre los cadáveres encontrados. Para los análisis estadísticos se usó el programa PAST versión 2.16 (Hammer, Harper \& Ryan, 2001). 
Variación temporal y espacial de los atropellos: La variación temporal de la tasa de atropellos se obtuvo al dividir el número total de cadáveres localizados en un mes en particular entre el número de kilómertos recorridos en dicho mes. Para determinar la distribución espacial de los atropellos, se dividió a la carretera en 74 tramos de $1 \mathrm{~km}$ y cada cadáver localizado se asignó al segmento que le correspondía por su ubicación. Se obtuvo el promedio $(\lambda)$ de cadáveres localizados por tramo. Dicho valor se usó luego para calcular la probabilidad de encontrar $x$ individuos muertos en cualquiera de esos segmentos $\mathrm{p}(x)$, bajo el supuesto de que la muerte de éstos ocurriera al azar a lo largo de la carretera. Si ese fuera el caso, el número de animales atropellados por segmentos de $1 \mathrm{~km}$ mostraría una distribución de Poisson, definida por: $\mathrm{p}(\mathrm{x})=\lambda^{\mathrm{x}} / \mathrm{x} ! \cdot \mathrm{e}^{\lambda}$ (Malo, Suárez \& Diez, 2004; Ascensão \& Mira, 2006). Los segmentos con número de animales atropellados por encima de lo que se esperaría por azar $(\mathrm{p}<0.05)$ se consideraron como "puntos negros".

Atropellos y tipo de cobertura terrestre: Se digitalizó con Arcview GIS 3.2 (Environmental System Research Institute, 1999) un mapa de cobertura a partir de una imagen de satélite Spot (654-332 del 28 de enero 2008) de tres bandas y resolución de $10 \mathrm{~m}$. Se reconocieron cinco tipos de cobertura: 1) áreas sub-urbanas, 2) bosques, 3) sabanas y potreros, 4) cultivos y 5) cuerpos de agua. Se realizaron reconocimientos en el terreno para localizar cuerpos de agua no previamente digitalizados y se agregaron al mapa de coberturas del suelo.

Se calculó la extensión de cada tipo de cobertura en franjas de $10 \mathrm{~km}(5 \mathrm{~km}$ a cada lado) y $200 \mathrm{~m}$ (100m a cada lado) de la vía. La superficie de cobertura en los "buffers" de $100 \mathrm{~m}$ de radio alrededor del punto de atropello se comparó (prueba de Mann-Whitney) con la encontrada en 100 localizaciones tomadas al azar a lo largo de la carretera. Estas últimas fueron seleccionadas entre los "track points" generados cada 15 segundos por el GPS durante un recorrido.
Cambios históricos: Con la finalidad de determinar si han cambiado las tasas de atropello de algunas especies, se contrastaron los resultados del presente estudio con los desarrollados en la década anterior por Pacheco (1993). Para ese análisis se tomó en cuenta que el señalado investigador incluyó a los animales localizados en el viaje de regreso. Esto produce un sesgo principalmente a favor de las aves, grupo que en su caso estuvo representado mayoritariamente $(90.7 \%)$ por individuos de especies diurnas. El sesgo debe ser mucho menor en el caso de los reptiles y los mamíferos, grupos que en la muestra de Pacheco (1993) estuvo conformada mayoritariamente por individuos de especies de hábitos nocturnos (74.4\% y $97.1 \%$, respectivamente). Es poco probable encontrar un animal nocturno recién atropellado en los viajes de vuelta. La determinación de si una especie es diurna o nocturna se basó en nuestro conocimiento de la fauna de los Llanos de Venezuela y en la información disponible en la literatura (Emmons, 1997; LaMarca \& Soriano, 2004).

Se realizaron comparaciones con otro estudio previo llevado a cabo en el área de estudio entre 1980 y 1982 (Ramo \& Busto, 1986), aunque con ciertas limitaciones, ya que estos autores no separaron los datos recolectados en la carretera Guanare-Guanarito de los tomados entre Guanare y Bruzual; tampoco indicaron para todas las especies el número de individuos localizados.

\section{RESULTADOS}

En los 26 recorridos realizados se localizaron 464 cadáveres: 66 aves ( 25 especies), 130 mamíferos (15 especies) y 268 reptiles (18 especies) (Apéndices I, II y III). Las curvas de rarefacción de especies (Fig. 1) tendieron a una asíntota en el caso de los mamíferos y los reptiles, pero no para las aves, que mostró una tendencia ascendente. Los individuos de las cinco especies más frecuentemente atropelladas (Leptodeira annulata, Didelphis marsupialis, Caiman crocodilus, Helicops angulatus 


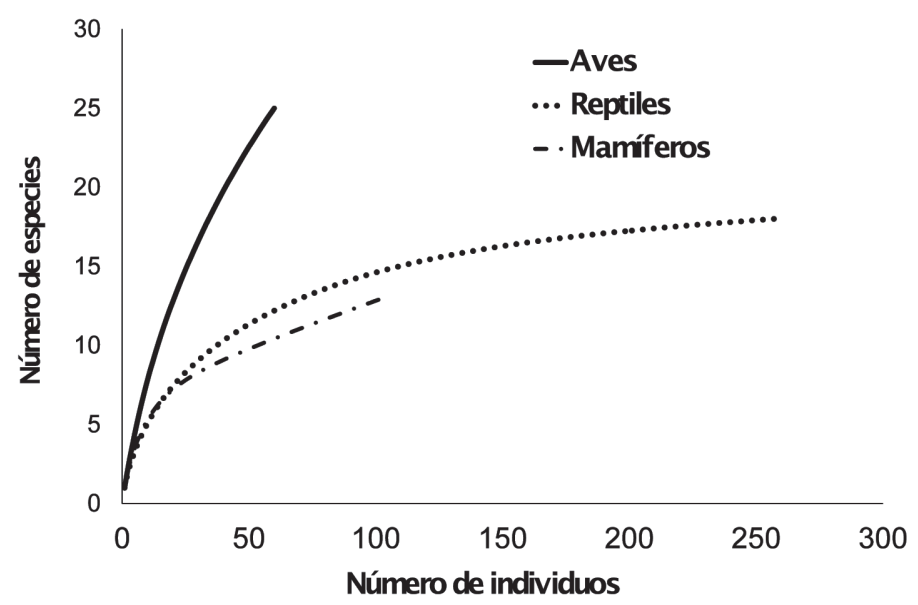

Fig. 1. Curvas de acumulación de especies a medida que el número de individuos atropellados localizados se incrementa. Fig. 1. Species accumulation curves as the number of road kills localized increases.

y Pseudoboa neuwiedii) representaron más de la mitad (51.5\%) de los cadáveres localizados. Las aves carroñeras y rapaces (órdenes Cathartiformes, Strigiformes, Falconiformes y Accipitriformes) representaron $48.5 \%$ de los cadáveres localizados para ese taxón.

Variación temporal y espacial: La frecuencia de atropellos mostró variaciones mensuales, con el mayor número de cadáveres localizados en abril y noviembre (Fig. 2). Estos son meses de transición entre las estaciones de sequía y lluvias. La predominancia de L. annulata es en gran parte responsable del patrón de distribución mensual que se observó, ya que esta serpiente presentó los máximos de atropellos en los mismos meses.

Sin incluir a los animales domésticos, para los 26 recorridos llevados a cabo se obtuvo un promedio de $5.93 \pm 3.45$ animales atropellados por tramo de $1 \mathrm{~km}$. Si las colisiones con vehículos se distribuyeran al azar a lo largo de la carretera, la probabilidad $\mathrm{p}(\mathrm{x})$, de encontrar 10 individuos muertos en un tramo de un $\mathrm{km}$

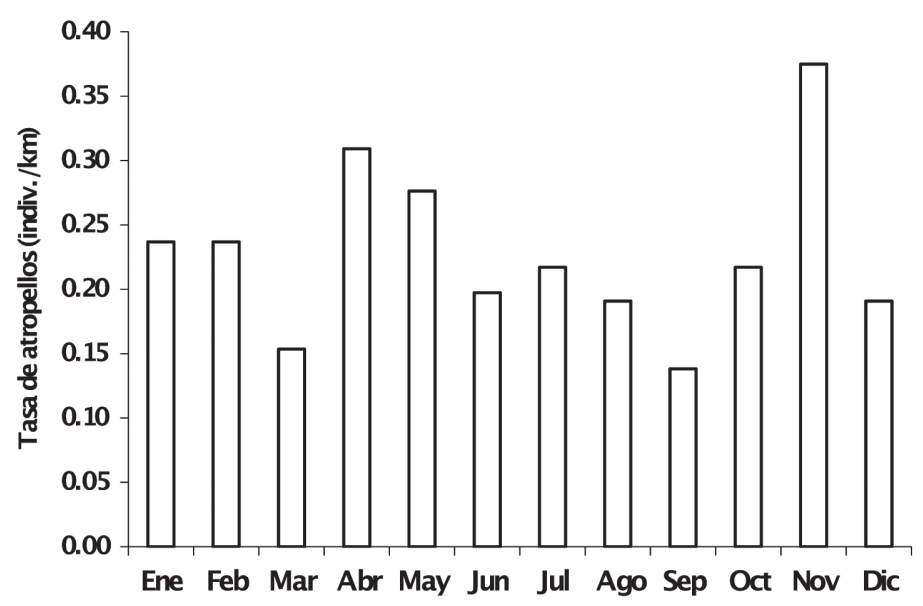

Fig. 2. Tasa de animales atropellados por mes en la carretera Guanare-Guanarito, Venezuela.

Fig. 2. Road kill rate per month found in the road Guanare-Guanarito, Venezuela. 
es baja $(\mathrm{p}<0.04)$; menos probable aún sería encontrar un número mayor que ese. No obstante, se encontraron 11 tramos con tasas de atropellos de 10 o más individuos (Fig. 3). Lo mismo se puede decir de tramos donde ocurrieron dos o menos colisiones $(\mathrm{p}<0.04)$ para lo cual se presentaron 12 casos. La distribución de los tramos de alta incidencia de atropellos ("puntos negros") mostró agrupamientos, con cuatro de esos segmentos desde el $\mathrm{km} 3$ al 7; cuatro tramos desde los $\mathrm{km} 18$ hasta el 25, y tres tramos consecutivos en los km 44, 45 y 46. Hubo mayor incidencia de atropellos por tramo entre Guanare y la Alcabala de Papelón (sitio intermedio entre los extremos de la carretera estudiada), que desde dicho punto hasta Guanarito (Kruskall-Wallis, $\chi^{2}=5.36, \mathrm{p}=0.021$ ).

Atropellos y tipo de cobertura terrestre: La importancia relativa de los tipos de cobertura del suelo varió entre las franjas a lo largo de la carretera (Cuadro 1). Así, por ejemplo, los bosques tuvieron una representación 2.8 veces mayor en la franja de $10 \mathrm{~km}$ que en la banda de 200m. Las proporciones de dichas coberturas fueron muy similares al comparar la franja más estrecha con los puntos tomados al azar.

Al considerar los distintos grupos de vertebrados, hubo diferencias, aunque no significativas, en la proporción de los distintos tipos de cobertura alrededor de los puntos de atropello y de los puntos tomados al azar, la más marcada de ellas fue para los mamíferos en la relación a la cobertura boscosa $(5.5 \%)$. Las cuatro especies para las que se calculó las mayores diferencias se muestran en la figura 4 . En ese gráfico, valores positivos o negativos indican que una determinada cobertura estuvo representada en mayor o menor proporción, respectivamente, alrededor de los puntos de atropello que alrededor de los puntos tomados al azar. Las diferencias fueron significativas para el caso de C. crocodilus en cuanto a los cuerpos de agua (Mann-Whitney $\mathrm{U}=1$ 317,

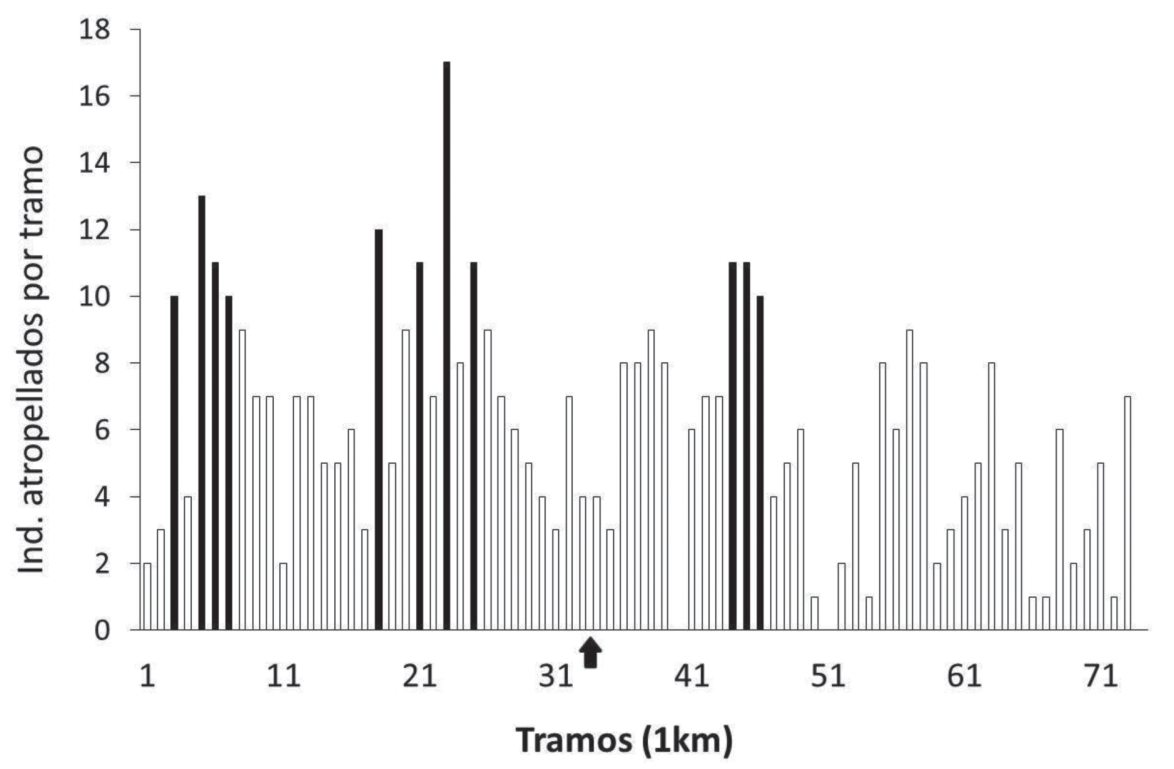

Fig. 3. Distribución espacial del número de animales silvestres atropellados en la carretera Guanare-Guanarito. Las barras en negro señalan los tramos de $1 \mathrm{~km}$ con alta incidencia de atropellos (11 o más individuos). La flecha marca la ubicación de la Alcabala de Papelón.

Fig. 3. Spatial distribution of the number of wildlife killed on the road Guanare-Guanarito. Black bars indicate the $1 \mathrm{~km}$ segments with high incidence of wildlife mortality (11 or more individuals). The arrow marks the location of the Papelón's checkpoint. 


\section{CUADRO 1}

Porcentajes de cobertura del suelo en franjas de $5 \mathrm{~km}$ y $100 \mathrm{~m}$ a cada lado de la carretera Guanare-Guanarito, así como en radios de $100 \mathrm{~m}$ alrededor de todos lugares de atropello de los individuos para cada grupo vertebrado $\mathrm{y}$ de 100 puntos tomados al azar

TABLE 1

Percentages of land cover in strips of $5 \mathrm{~km}$ and $100 \mathrm{~m}$ on either side of the road-Guanare-Guanarito and in $100 \mathrm{~m}$ radius around all places collision of individuals for each vertebrate group and 100 points at random

\begin{tabular}{lcccccc}
\multicolumn{1}{c}{ Cobertura } & $5 \mathrm{~km}$ & $100 \mathrm{~m}$ & Aves & Mamíferos & Reptiles & \multicolumn{2}{c}{ Puntos al azar } \\
Bosques & 27.3 & 9.8 & 10.7 & 15.2 & 11.8 & 9.7 \\
Área urbanizada & 3.8 & 1.3 & 0.7 & 0.5 & 2.5 & 1.4 \\
Cuerpos de agua & 0.4 & 2.2 & 1.1 & 2.6 & 2.6 & 2.9 \\
Cultivos & 8.3 & 0.8 & 1.6 & 0.3 & 1.5 & 1.0 \\
Sabanas y potreros & 60.2 & 86.0 & 85.8 & 81.3 & 81.6 & 85.0 \\
\hline
\end{tabular}

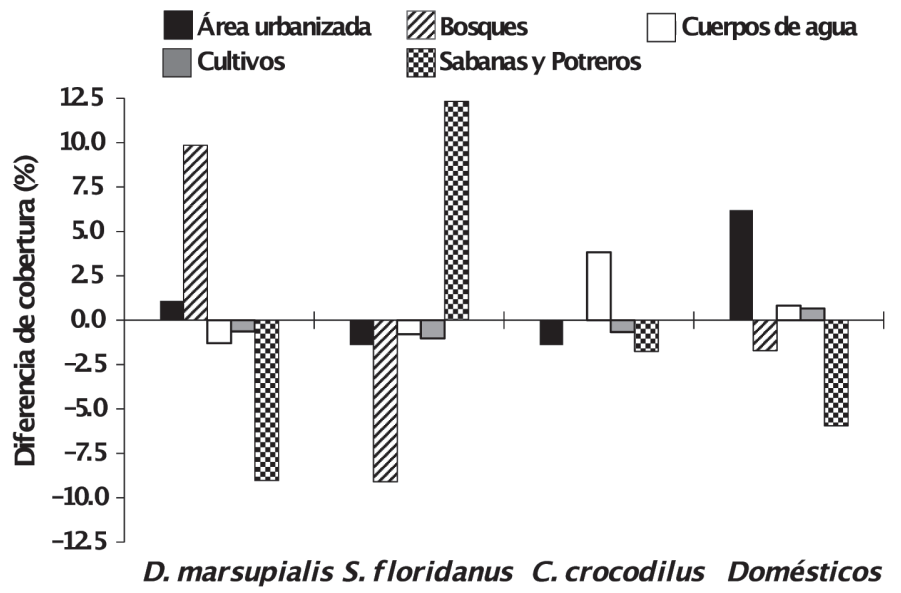

Fig. 4. Diferencias entre los porcentajes de cobertura en $100 \mathrm{~m}$ de radio alrededor de todos los puntos de atropellos de algunas especies y los de radios de la misma extensión alrededor de 100 puntos tomados al azar a lo largo de la carretera Guanare-Guanarito, Venezuela. "Domésticos" incluye a perros y gatos.

Fig. 4. Differences between the percentages of coverage in 100m-radii around all points of vehicle-collision of some species, and those percentages in radii of the same size around 100 points at random along the road Guanarito-Guanare, Venezuela. "Domésticos" includes dogs and cats.

$\mathrm{p}=0.047)$; de $D$. marsupialis en relación a los bosques ( $U=1507, p=0.022)$, y de los animales domésticos respecto a la cobertura urbana $(\mathrm{U}=1$ 004, $\mathrm{p}<0.01)$. No obstante que las diferencias más marcadas en términos porcentuales se encontraron para $S$. floridanus, estas no fueron significativas, ni para los bosques ni para las sabanas y potreros $(\mathrm{p}>0.19)$.

Comparaciones históricas: Los reptiles fue el grupo con más cadáveres localizados, seguido por los mamíferos y luego las aves.
Las diferencias en el número de individuos por grupo con respecto a los que encontró Pacheco (1993) fueron altamente significativas $\left(\chi^{2}=38.2, \mathrm{p}<0.001,2 \mathrm{gl}\right)$. Por otra parte, en ambas investigaciones las aves fueron el grupo representado por más especies, seguido por los mamíferos y luego los reptiles.

La proporción de animales nocturnos fue de $25.0 \%$ en el caso de las aves y $89.5 \%$ y $99.0 \%$ para reptiles y mamíferos, respectivamente. Las diferencias en frecuencia con respecto a las calculadas a partir de los datos 
en Pacheco (1993) fueron significativas para las aves $\left(X^{2}=9.36, \mathrm{p}=0.02\right)$ y para los reptiles $\left(X^{2}=20.36, \mathrm{p}<0.001\right)$ pero no para los mamífe$\operatorname{ros}\left(X^{2}=1.13, \mathrm{p}=0.44\right)$.

La TA del presente estudio $(0.2282 \pm 0.113$ indiv. $/ \mathrm{km})$ fue $28.3 \%$ superior a la calculada a partir de los datos de Pacheco (1993) (0.1778indiv./km). Al considerar a cada grupo de vertebrado por separado, la tasa de mortalidad para los mamíferos $(0.0546 \pm 0.039 \mathrm{indiv} . / \mathrm{km})$ es prácticamente idéntica a la de Pacheco (1993) (0.0564indiv./ $\mathrm{km})$. En el caso de los reptiles, la TA del presente estudio $(0.1393 \pm 0.088 \mathrm{indiv} . / \mathrm{km})$ fue $84.6 \%$ mayor que la de Pacheco (1993) (0.0755indiv./ $\mathrm{km})$; para las aves esas tasas pasaron de
$0.0459 \mathrm{indiv} . / \mathrm{km}$ a $0.0343 \pm .022 \mathrm{indiv} . / \mathrm{km}$, un decrecimiento de $-25.3 \%$. Algunas especies mostraron incrementos considerables $(H$. angulatus, $P$. neuwiedii, $M$. tridactyla), mientras que otras decrecieron, como por ejemplo C. carinatus, I. iguana, T. teguixin, Columbina spp. у $C$. thous (Cuadro 2).

\section{DISCUSIÓN}

En la presente investigación, al igual que en las de Ramo \& Busto (1986) y Pacheco (1993), los reptiles fueron el grupo con mayor número de individuos atropellados, seguido por los mamíferos y luego las aves. Esta secuencia es muy variable en diversos estudios realizados

\section{CUADRO 2}

Comparación de número de individuos y tasa de atropellos (TA, indiv./km) del presente estudio con los de Pacheco (1993). La última columna muestra las diferencias en las tasas de atropellos en términos porcentuales

TABLE 2

Comparison of number of individuals and roadkills rate (TA, indiv./km) of this study with those of Pacheco (1993). Last column shows changes in collision rates as percentages

\begin{tabular}{|c|c|c|c|c|c|}
\hline Especie & $\begin{array}{l}\text { No. individuos } \\
\text { Presente estudio } \\
(2008-2010)\end{array}$ & $\begin{array}{l}\text { No. individuos } \\
\text { Pacheco } 1993 \\
(1991-1992)\end{array}$ & $\begin{array}{c}\text { TA presente } \\
\text { estudio }\end{array}$ & TA Pacheco 1993 & Dif. (\%) \\
\hline \multicolumn{6}{|l|}{ Reptiles } \\
\hline Boa constrictor & 5 & 16 & 0.0026 & 0.0043 & -40 \\
\hline Caiman crocodilus & 33 & 55 & 0.0172 & 0.0148 & 16 \\
\hline Chironius carinatus & 4 & 18 & 0.0021 & 0.0048 & -57 \\
\hline Epicrates maurus & 6 & 21 & 0.0031 & 0.0056 & -45 \\
\hline Helicops angulatus & 25 & 1 & 0.0130 & 0.0003 & 4739 \\
\hline Iguana iguana & 4 & 14 & 0.0021 & 0.0038 & -45 \\
\hline Leptodeira annulata & 119 & 74 & 0.0619 & 0.0199 & 211 \\
\hline Pseudoboa neuwiedii & 23 & 7 & 0.0120 & 0.0019 & 536 \\
\hline Tupinambis teguixin & 1 & 15 & 0.0005 & 0.0040 & -87 \\
\hline \multicolumn{6}{|l|}{ Aves } \\
\hline Columbina spp. & 3 & 32 & 0.0016 & 0.0086 & -82 \\
\hline Crotophaga ani & 3 & 15 & 0.0016 & 0.0040 & -61 \\
\hline Buhos y lechuzas (Strigiformes) & 11 & 14 & 0.0057 & 0.0038 & 52 \\
\hline \multicolumn{6}{|l|}{ Mamíferos } \\
\hline Cerdocyon thous & 9 & 61 & 0.0047 & 0.0164 & -71 \\
\hline Dasypus novemcinctus & 13 & 8 & 0.0068 & 0.0021 & 215 \\
\hline Didelphis marsupialis & 39 & 74 & 0.0203 & 0.0199 & 2 \\
\hline Myrmecophaga tridactyla & 3 & 1 & 0.0016 & 0.0003 & 481 \\
\hline Procyon cancrivorus & 9 & 8 & 0.0047 & 0.0021 & 118 \\
\hline Sylvilagus floridanus & 11 & 5 & 0.0057 & 0.0013 & 326 \\
\hline Tamandua tetradactyla & 13 & 24 & 0.0068 & 0.0064 & 5 \\
\hline
\end{tabular}


en el Neotrópico. Las aves fueron el grupo predominante en los estudios de Milli \& Passamani (2006) y Turci \& Bernarde (2009); los reptiles predominaron en los trabajos de Hengemühle \& Candemartori (2008) y Quintero-Ángel et al. (2012); los mamíferos destacaron en MongeNájera (1996), Rosa \& Mauhs (2004), Coelho et al. (2008), Cunha et al. (2010), Menenguetti, Menenguetti, \& Trevisan (2010), y Santana (2012). Esos contrastes se explican por las disímiles características (altitud, latitud, clima, tipos de hábitats, composición faunística) de las áreas de estudio y también por las distintas metodologías y enfoques empleados por los investigadores.

Las diferencias en metodologías, características geográficas y ecológicas de las áreas de estudio y tipo de carreteras consideradas, hacen difícil la comparación de las tasas de atropellos entre distintas investigaciones realizadas en el Neotrópico, incluso de aquellas realizadas en el mismo país (Cunha et al., 2010). Por lo demás, muchos investigadores no reportan esas cifras o lo hacen en unidades diferentes, lo que obliga a calcularlas a partir de los datos aportados.

Para ilustrar lo anteriormente dicho, la tasa de atropello de las aves del presente estudio $(0.0343$ indiv. $/ \mathrm{km})$ fue muy semejante a la obtenida por Melo \& Santos-Filho (2007) (0.0349indiv. $/ \mathrm{km})$ y superior a la obtenida por Cunha et al. (2010) (0.0105indiv./km). La tasa obtenida para los mamíferos (0.0546indiv. $/ \mathrm{km})$ estuvo cercana a la de Melo \& Santos-Filho (2007) (0.0794indiv./km), pero fue muy superior a la de Cunha et al. (2010) (0.0122indiv./ $\mathrm{km})$. En el caso de los reptiles, la tasa de atropello (0.1393indiv. $/ \mathrm{km})$ se puede considerar muy alta si se compara con las de MongeNájera (1996) (0.0015).

La periodicidad de atropellos a lo largo del año está asociada a la fenología de las especies, la cual está a su vez determinada principalmente por los cambios en el régimen lluvioso en el transcurso del año (Rodda, 1989; Ashley \& Robinson, 1996; Clevenger et al., 2003; Gumier-Costa \& Sperber, 2009; Lagos, Picos, \& Valero, 2012). Los picos observados en el presente estudio se explican porque algunas especies, como por ejemplo C. crocodilus, aumentan su movilidad al inicio de las lluvias (abril) o al comienzo de la época seca (noviembre) (Ramo \& Busto, 1986).

Hubo tramos de la carretera donde la TA excedió la esperada al azar (puntos negros), lo cual ha sido mostrado en otros estudios (Clevenger et al., 2003; Malo et al., 2004; Santos et al., 2007; Coelho et al., 2008). La mayor frecuencia de puntos negros en el trayecto que va de Guanare a la alcabala de Papelón se explica por: 1) la mayor intensidad de tráfico en dicho trayecto; 2) el mal estado de la carretera en el segundo trayecto, que obliga a conducir a baja velocidad en varios lugares, en los cuales se localizaron, por cierto, los menores números de animales atropellados, y 3) los recorridos siempre se iniciaron en Guanare, de manera que en los últimos kilómetros siempre eran recorridos una hora o más después del inicio, cuando parte de los animales atropellados ya habrían sido removidos por las aves carroñeras y rapaces. La relevancia de este último factor queda de manifiesto cuando se considera que $51.5 \%$ de las aves atropelladas pertenecen a esos grupos.

El tipo de cobertura a lo largo de la carretera seguramente influyó en las especies y en el número de individuos atropellados, como se comprobó para D. marsupialis con los bosques, los mamíferos domésticos con las áreas urbanas y $C$. crocodilus con los cuerpos de agua. La aparente asociación de $S$. floridanus con sabanas y potreros no pudo ser comprobada estadísticamente, quizás por el pequeño tamaño de la muestra. Además de un problema de escala a la cual se trabaja, hay que considerar aquellos factores no evaluados en este estudio que pueden incidir en la frecuencia y ubicación exacta donde ocurre el atropellamiento; entre ellos podríamos mencionar la periodicidad de los muestreos, la visibilidad del cadáver, el volumen de tráfico, la abundancia y diversidad de los carroñeros (Santos et al., 2007), entre otros.

Comparaciones históricas: El incremento de la tasa de mortalidad con respecto a la calculada con los datos de Pacheco (1993) fue de $28.3 \%$, menor a lo que se esperaría si 
se considera que se calculó un incremento de $64.5 \%$ en el flujo de vehículos para el lapso transcurrido entre los estudios. Esta última cifra es seguramente una subestimación, si se toma en cuenta que el parque automotor del país se incrementó en $113 \%$ en el lapso 19982008 (Ramírez, 2012). Por otra parte, no necesariamente hay una relación lineal (creciente o decreciente) entre volumen de tráfico y las colisiones de vehículos con fauna silvestre. Pudiera haber un umbral de tráfico por encima del cual ya algunas especies eviten la carretera (Fahrig, Neill, \& Duquesnel, 2001; Grilo, Bissonette, \& Santos-Reis, 2008), lo que se reflejaría en la tasa general de mortalidad, dando la impresión errónea de que el tamaño de las poblaciones ha cambiado.

La magnitud y sentido del cambio en la TA con respecto a Pacheco (1993) no fue el mismo en todos los grupos vertebrados. Por el contrario, hubo un estancamiento en el caso de los mamíferos, un aumento para los reptiles, mientras que para las aves ésta se redujo (-25.3\%). Dentro de cada grupo hubo especies para las que aumentó y otras en que disminuyó la TA, por lo que analizaremos algunos casos.

Entre las especies con marcado descenso en sus tasas de atropello se encuentran los reptiles B. constrictor, E. maurus, I. iguana y C. carinatus. Todas estas especies están asociadas a ambientes boscosos (Rodda, 1989; La-Marca \& Soriano, 2004). La fragmentación y pérdida de bosques en Venezuela ha sido particularmente acentuada en los Llanos Occidentales (Pacheco-Angulo et al., 2011), precisamente donde se localiza la carretera Guanare-Guanarito. Se podría añadir en favor de la hipótesis de que las poblaciones de algunas especies han declinado a medida que avanza la deforestación, que en el estudio de Ramo \& Busto (1986) la iguana ocupó el primer lugar entre los reptiles $(39.6 \%)$ y C. carinatus fue la serpiente más atropellada. En el caso de I. iguana y C. carinatus, que son especies de hábitos diurnos, se podría argumentar que la disminución en la TA es un artefacto producido por el hecho de que Pacheco (1993) incluyó en sus muestreos a los animales observados en los viajes de regreso. Ya se señaló que en ese tipo de recorridos es poco probable localizar cadáveres de animales recién atropellados, como se comprobó en los 26 muestreos realizados en la presente investigación, en los que sólo se observó un animal muerto en el viaje de retorno (precisamente, una iguana).

Fahrig et al. (2001) argumentaron que las muertes de animales en la carretera son análogas a las "capturas por unidad de esfuerzo" donde la "captura" es el número de animales atropellados y el "esfuerzo" es una función directa del tráfico vehicular. Ante un incremento en el tráfico, una disminución (o estancamiento) en la TA se interpreta como que ha ocurrido un decrecimiento de la población de la especie analizada. De acuerdo con esos criterios, se puede concluir que las poblaciones de los reptiles señalados en el párrafo anterior declinaron en el área de estudio.

El rabipelado (D. marsupialis) ha sido el mamífero más atropellado en los estudios hasta ahora realizados en la carretera GuanareGuanarito, pero la TA se redujo levemente en la última década (2\%). Dado el incremento en el flujo de vehículos se puede concluir que su población también declinó, aunque no en la magnitud de los reptiles señalados anteriormente. El rabipelado ocupa ambientes boscosos, pero su adaptación a ambientes intervenidos y urbanizados (Cordero \& Barque, 1992; MongeNájera, 1996; Cáceres, 2000) pudiera haber disminuido el impacto que significaría para esta especie la pérdida de cobertura boscosa.

La explicación para la aparente disminución de las poblaciones de palomitas (Columbina spp.), quizás se encuentre en que la importancia de los cultivos de cereales, especialmente del sorgo, fue menor durante el presente estudio que durante los años en que trabajó Pacheco (1993). Estas palomitas bajan a la carretera para comer los granos que caen de los camiones que transportan maíz y sorgo.

Así como algunas especies de ambientes boscosos habrían disminuido su población, otras de ambientes de sabana mostraron un incremento en las tasas de atropellos. En esta situación tenemos a las serpientes $L$. annulata 
y $P$. neuwiedii y al conejo $S$. floridanus. La primera de estas especies no se encuentra entre los reptiles más atropellados reportados por Ramo \& Busto (1986). Dada la magnitud de los cambios experimentados, pudiera interpretarse como que ocurrió un aumento en sus poblaciones.

El más notable incremento en la TA resultó para $H$. angulatus. Ello parece indicar la progresiva invasión de esta especie a un área no previamente ocupada, o que esta serpiente ha respondido lentamente ante la presencia de un hábitat favorable (los préstamos), creados en 1972. Esta serpiente no aparece en la lista de animales atropellados de Ramo \& Busto (1986).

La disminución de las tasas de atropellos del zorro (C. thous) y del mato ( $T$. teguixin) fueron las más acentuadas entre los mamíferos y los reptiles, respectivamente. El zorro ocupó el segundo lugar en frecuencia de atropellos en los estudios de Ramo \& Busto (1986) y Pacheco (1993). Ha sido además el mamífero con más cadáveres localizados en otros estudios realizados en su área de distribución (Cherem et al., 2007; Melo \& Santos-Filho, 2007; Cáceres et al., 2010; Nobrega de Sousa \& Cordeiro de Miranda, 2010; Cáceres, Casella, \& Goulart, 2012; Hegel et al., 2012). Especies del género Tupinambis, por su parte, se encuentran en el primer lugar de la lista de reptiles atropellados en algunas localidades estudiadas de Brasil (Rosa \& Mauhs, 2004; Santana, 2012). Habría que realizar nuevos estudios para buscar posibles explicaciones para la aparente disminución poblacional de esas especies en la carretera Guanare-Guanarito.

Especies de atención prioritaria: En el presente estudio se localizaron tres $M$. tridactyla atropellados. Pacheco (1993) en 49 recorridos sólo localizó un ejemplar de esta especie y Ramo \& Busto (1986) no reportaron ninguno para sus 31 viajes Guanare-Guanarito. Esta mayor tasa de mortalidad podría indicar un aumento en la población de osos hormigueros, quizás favorecido por la deforestación para crear potreros para el ganado vacuno, o podría deberse al mayor flujo de vehículos, algo preocupante si tomamos en cuenta que esta especie es considerada como vulnerable (Rodríguez \& Rojas-Suárez, 2008). Los atropellamientos son considerados precisamente, como una de las principales amenazas para $M$. tridactyla (Ojasti \& Lacabana, 2008; Cunha et al., 2010; Miranda \& Meritt, 2013).

Otra especie que mostró una tasa de mortalidad preocupante fue $T$. tetradactyla. Individuos del género Tamandua aparecen de manera destacada en los estudios de fauna atropellada en el Neotrópico (Monge-Nájera, 1996; Fischer et al. 2004; Pinowski, 2005; Pereira, Andrade \& Fernandes, 2006; Melo \& Santos-Fihlo, 2007; Gumier-Costa \& Sperber, 2009; Cunha et al., 2010). Es muy probable que los atropellos sean la principal causa de mortalidad para T. tetradactyla en Venezuela. Aún cuando esta especie está incluida en la categoría de "Riesgo Menor" por la UICN, dicha institución reconoce que la pérdida de hábitat, los incendios de vegetación y tráfico en las carreteras, pudieran representar amenazas en ciertas áreas (Miranda \& Merrit, 2013), por lo que se recomienda realizar estudios sobre esta especie.

Medidas de manejo: El sistema vial de Venezuela ha sido, desde hace tiempo, reputado como uno de los mejores de Latinoamérica (Meyer de Shauensee \& Phelps-Jr, 1978). No existen, sin embargo, políticas públicas que obliguen a los entes encargados de la construcción de carreteras a implementar medidas de mitigación del impacto directo que generan sobre la fauna silvestre. En países como Brasil esas políticas son de muy reciente data (Fischer et al., 2004).

Por cuestión de costos, es en los puntos negros donde se suelen implementar mecanismos para mitigar la mortalidad de la fauna. Esta medida, sin embargo, tiende a proteger principalmente a las especies muy numerosas ( $L$. annulata y D. marsupialis, por ejemplo) que no son necesariamente las que requieren mayor atención. Bager \& Rosa (2010) proponen establecer prioridades de protección de acuerdo a índices basados en: 1) la riqueza de especies, 
2) diversidad de especies, 3) tasa de atropellos de especies focales y, 4) presencia de especies amenazadas. Se recomienda más estudios para intentar enfoques como el señalado.

Respecto a las medidas para mitigar el impacto de la carretera sobre la fauna, nos limitamos a replantear las recomendaciones de Ramo \& Busto (1986) y Pacheco (1993): 1) educar a los conductores con folletos divulgativos sobre la problemática, 2) colocar vallas y avisos, principalmente en los "puntos negros" para alertar a los conductores sobre el paso de fauna y la necesidad de que reduzcan la velocidad; los avisos deben ser visibles de noche, que es cuando ocurren la mayor parte de los atropellamientos, 3) mantener permanentemente una franja libre de vegetación en los bordes de la carretera para dar mayor oportunidad a los conductores y la fauna de evitar los atropellamientos. Esperamos que esta contribución estimule la investigación sobre la problemática de la fauna atropellada tanto en Venezuela como en el resto de los países del trópico americano.

\section{AGRADECIMIENTOS}

Para el trabajo de campo se contó con la asistencia de Luis Sánchez, Pedro Carrillo Seijas, Richard Narváez, Alcira y José Velázquez, Miguel Fernando Zapata y Wilfredo Ochoa, quienes colaboraron en la obtención de los datos, y ayudaron a identificar las especies observadas durante los recorridos.

\section{RESUMEN}

Uno de los aspectos más investigados en relación a la "ecología de la carretera" es el atropellamiento de vertebrados silvestres. Se evaluó la distribución espacial y temporal de los atropellos de vertebrados en la carretera Guanare-Guanarito, estado Portuguesa, Venezuela. Desde 2008 hasta el 2010 se realizaron 26 viajes en carro a lo largo de $74 \mathrm{~km}$, a $50-60 \mathrm{~km} / \mathrm{h}$, para un total recorrido de 1 $924 \mathrm{~km}$. Se encontraron 464 animales muertos: 66 aves $(25$ especies), 130 mamíferos (15 especies) y 268 reptiles (18 especies). La serpiente Leptodeira annulata $(\mathrm{n}=119)$, el rabipelado Didelphis marsupialis $(\mathrm{n}=39)$ y la baba Caiman crocodilus $(\mathrm{n}=33)$ fueron las especies con mayor frecuencia de atropellos. Excluyendo a los animales domésticos, se

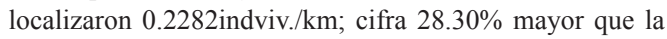

reportada en estudios previos en la misma vía. Los cambios en la frecuencia relativa de atropellos de algunas especies con respecto a los reportados hace 20 años, se relacionan con el incremento en el flujo vehicular y por modificaciones en el uso de la tierra. Se identificaron segmentos donde el número de individuos atropellados supera al esperado por azar. Los atropellos pudieran ser la principal causa de mortalidad para especies como el oso melero (Tamandua tetradactyla) y el oso hormiguero (Myrmecophaga tridactyla), esta última considerada como una especie vulnerable. Se recomiendan algunas medidas básicas para disminuir la mortalidad de fauna en la carretera.

Palabras clave: fauna atropellada, conservación de fauna, Llanos de Venezuela, Leptodeira annulata, Myrmecophaga tridactyla.

\section{REFERENCIAS}

Andrews, K. M., \& Gibbons, J. W. (2005). How do highways influence snake movement? behavioral responses to roads and vehicles. Copeia, 2005, 4, 772-782.

Arévalo, J. E., \& Newhard, K. (2011). Traffic noise affects forest bird species in a protected tropical forest. Journal of Tropical Biology, 59(2), 969-980.

Ascensão, F., \& Mira, A. (2006). Spatial patterns of road kills: a case study in southern Portugal. In C. L. Irwin, P. Garrett \& K. P. McDermott (Eds.). Proceedings of the 2005 International Conference on Ecology and Transportation, Center for Transportation and the Environment (pp. 641-646). North Carolina State University, Raleigh, NC: Center for Transportation and the Environment.

Ashley, E. P., \& Robinson, J. T. (1996). Road mortality of amphibians, reptiles and other wildlife on the Long Point Causeway, Lake Erie, Ontario. The Canadian Field-Naturalist, 110, 403-412.

Aymard, J. G., Farreras, J., \& Schargel, R. (2011). Bosques secos macrotérmicos de venezuela. Biollania, 10, 155-177.

Bager, A., \& Rosa, C. A. (2010). Priority ranking of road sites for mitigating wildlife roadkill. Biota Neotropica, 10(4), 149-153.

Cáceres, N. C. (2000). Population ecology and reproduction of the white-eared oppossum Didelphis albiventris (Mammalia, Marsupialia) in an urban environment of Brazil. Ciencia e Cultura, 52(3), 171-174.

Cáceres, N. C., Hannibal, W., Freitas, D. R., Silva, E. L., Roman, C., \& Casella, J. (2010). Mammal occurrence and roadkill in two adjacent ecoregions (Atlantic Forest and Cerrado) in south-western Brazil. Zoologia, 27(5), 709-717.

Cáceres, N. C., Casella, J., \& Goulart, C. S. (2012). Variaçao espacial e sazonal de atropelamentos de mamíferos 
no bioma cerrado, rodovia BR 262, Sudoeste do Brasil. Mastozoología Neotropical, 19(1), 21-33.

Carr, L. W., Fahrig, L., \& Pope, S. E. (2002). Impacts of landscape transformation by roads. In K. J. Gutzwiller (Ed.). Applying landscape ecology in biological conservation (pp. 225-243). New York: SpringerVerlag New York, Inc.

Case, R. M. (1978). Interstate highway road-killed animals: a data source for biologists. Wildlife Society Bulletin, 6(1), 8-13.

Cherem, J. J., Kammers, M., Ghizoni-Jr, I. R., \& Martins, A. (2007). Mamíferos de médio e grande porte atropelados em rodovias do Estado de Santa Catarina, sul do Brasil. Biotemas, 20(2), 81-96.

Clevenger, A. P., Chruszcz, B., \& Gunson, K. E. (2003). Spatial patterns and factors influencing small vertebrate fauna road-kill aggregations. Biological Conservation, 109, 15-26.

Coelho, I. P., Kindel, A., \& Coelho, A. V. P. (2008). Roadkills of vertebrate species on two highways through the Atlantic Forest Biosphere Reserve, southern Brazil. European Journal of Wildlife Research, 54, 689-699. doi: 10.1007/s10344-008-0197-4

Coffin, A. W. (2007). From roadkill to road ecology: A review of the ecological effects of roads. Journal of Transport Geography, 15, 396-406. doi:10.1016/j. jtrangeo.2006.11.006

Cordero, G. A., \& Barque, N. (1992). Feeding habits of the opossum (Didelphis marsupialis) in natural and urban environments in Venezuela. Acta Cientifica Venezolana, 43(3), 159-163.

Cunha, H. F., Moreira, F. G. A., \& Silva, S. S. D. (2010). Roadkill of wild vertebrates along the GO-060 road between Goiânia and Iporá, Goiás State, Brazil. Acta Scientiarum. Bological Sciences, 32(3), 257-263.

Delgado, C. A. (2007). Muerte de mamíferos por vehículos en la vía del Escobero, Envigado (Antioquía), Colombia. Actualidad Biológica, 29(87), 229-233.

Emmons, L. H. (1997). Neotropical rainforest mammals. Chicago: The University of Chicago Press.

Fahrig, L., Neill, K. E., \& Duquesnel, J. G. (2001). Interpretation of joint trends in traffic volume and trafficrelated wildlife mortality: a case study from Key Largo, Florida. Florida: Road Ecology Center, John Muir Institute of the Environment, UC Davis.

Fischer, W. A., Ramos-Neto, M. B., Silveira, L., \& Jacomo, A. T. (2004). Human transportation network as ecological barrier for wildlife on Brazilian PantanalCerrado corridors. In C. L. Irwin, P. Garrett \& K. P. McDermott (Eds.). Proceedings of the 2005 International Conference on Ecology and Transportation, Center for Transportation and the Environment (pp. 182-194). North Carolina State University, Raleigh, NC: Center for Transportation and the Environment.
Forman, R. T. T., \& Alexander, L. E. (1998). Roads and their major ecological effects. Annual Review of Ecology and Systematics, 29, 207-231.

Frías, O. (1999). Estacionalidad de los atropellos de aves en el centro de España: Número y edad de los individuos y riqueza y diversidad de especies. Ardeola, 46(1), 23-30.

Fundación para el Desarrollo de la Región Centro Occidental. (2004). Dossiers municipios Guanare, Guanarito y Papelón. FUDECO. Barquisimeto, Venezuela. Disponible en: http://www.fudeco.gob.ve/dossier_docs. php.

Gehrt, S. D., Hubert, G. F., \& Ellis, J. A. (2002). Long-term population trends of raccoons in Illinois. Wildlife Society Bulletin, 30, 457-463.

Glista, D. J., \& DeVault, T. L. (2008). Road mortality of terrestrial vertebrates in Indiana. Proceeding of the Indiana Academy of Science, 117(1), 55-62.

Grilo, C., Bissonette, J. A., \& Santos-Reis, M. (2008). Response of carnivores to existing highway culverts and underpasses: implications for road planning and mitigation. Biodiversity Conservation, 17, 1685-1699. doi: 10.1007/s10531-008-9374-8

Grilo, C., Bissonette, J. A., \& Santos-Reis, M. (2009). Spatial-temporal patterns in Mediterranean carnivore road casualties: Consequences for mitigation. Biological Conservation, 142, 301-313. doi:10.1016/j. biocon.2008.10.026

Gumier-Costa, F., \& Sperber, C. F. (2009). Atropelamentos de vertebrados na Floresta Nacional de Carajás, Pará, Brasil. Acta Amazonica, 39(2), 1-10.

Hammer, O., Harper, D. A. T., \& Ryan, P. D. (2001). PAST: Paleontological statisctics software package for education and data analysis. Paleontología Electronica, 4(1), 1-9.

Hegel, C. G. Z., Consalter, G. C., \& Zanella, N. (2012). Mamíferos silvestres atropelados na rodovia RS-135, norte do Estado do Rio Grande do Sul. Biotemas, 25(2), 165-170.

Hengemühle, A., \& Cademartori, C. V. (2008). Levantamento de mortes de vertebrados silvestres devido a atropelamento em un trecho da estrada do mar (RS389). Biodiversidade Pampeana, 6(2), 4-10.

Lagos, L., Picos, J., \& Valero, E. (2012). Temporal pattern of wild ungulate-related traffic accidents in northwest Spain. European Journal of Wildlife Research, 58, 661-668. doi: 10.1007/s10344-012-0614-6

La-Marca, E., \& Soriano, P. J. (2004). Reptiles de los Andes de Venezuela. Mérida, Venezuela: Fundación Polar, Conservación Internacional, CODEPRE-ULA, Fundacite Mérida, Biogeos.

Madsen, A. B., Strandgaard, H., \& Prang, A. (2002). Factors causing traffic killings of roe deer Capreolus capreolus in Denmark. Wildlife Biology, 8(1), 55-61. 
Malo, J. E., Suárez, F., \& Díez, A. (2004). Can we mitigate animal-vehicle accidents using predictive models? Journal of Applied Ecology, 41, 701-710.

Melo, E. S., \& Santos-Filho, M. (2007). Efeitos da BR-070 na Província Serrana de Cáceres, Mato Grosso, sobre a comunidade de vertebrados silvestres. Revista Brasileira de Zoociencias, 9(2), 185-192.

Menenguetti, D. U. O., Menenguetti, N. F. S. P., \& Trevisan, O. (2010). Georreferenciamiento e reavaliacao da mortalidade de animais silvestres na linha 200 entre os municipios de Ouro Preto e Vale do ParaisoRO. Revista Cientifica da Facultade de Educaçao e Meio Ambiente, 1, 58-64.

Meyer de Schauensee, R., \& Phelps-Jr., W. H. (1978). A guide to the birds of Venezuela. New Jersey: Princeton University Press.

Milli, M. S., \& Passamani, M. (2006). Impacto da Rodovia Josil Espíndula Agostini (ES-259) sobre a mortalidade de animais silvestres (Vertebrata) por atropelamento. Natureza on line, 4(2), 40-46. Disponible en: http://www.naturezaonline.com.br

Miranda, F., \& Meritt Jr., D. A. (2013, Enero 13). Tamandua tridactyla. IUCN Red List of Threatened Species. Version 2012.2. Disponible en: http://www.iucnredlist.org

Monge-Nájera, J. (1996). Vertebrate mortality on tropical highways: The Costa Rica case. Vida Silvestre Neotropical, 5(2), 154-156

Nobrega de Sousa, M. A., \& Cordeiro de Miranda, P. (2010). Mamíferos terrestres encontrados atropelados na rodavia BR-230/BP entre Campina Grande e Joao Pessoa. Biofar, 4(2), 72-82.

Ojasti, J., \& Lacabana, P. (2008). Oso hormiguero Myrmeecophaga tridactyla. In J. P. Rodríguez \& F. Rojas Suárez (Eds.). Libro Rojo de la fauna venezolana. Caracas, Venezuela: Provita y Shell de Venezuela, S.A.

Pacheco-Angulo, C., Aguado-Suárez, I., \& Mollicone, D. (2011). Dinámica de la deforestación en Venezuela: análisis de los cambios a partir de mapas históricos. Interciencia, 36(8), 578-586.

Pacheco, P. (1993). Evaluación de la mortalidad de fauna silvestre por atropellamiento en la carretera Guanare-Guanarito, estado Portuguesa. Trabajo de Aplicación de Conocimientos, Vicerrectorado de Producción Agrícola. Programa Recursos Naturales Renovables, Universidad Nacional Experimental de los Llanos (UNELLEZ), Guanare, Venezuela. Disponible en http://dl.dropbox.com/u/90486101/Pacheco Maica 1993.pdf

Pereira, A. P. F. G., Andrade, F. A. G., \& Fernandes, M. E. B. (2006). Dois anos de monitoramento dos atropelamentos de mamíferos na rodovia PA-458, Bragança, Pará. Boletim do Museu Paraense Emilio Goeldi, 1(3), 77-83.
Pinowski, J. (2005). Roadkills of vertebrates in Venezuela. Revista Brasilera de Zoologia, 22(1), 191-196.

Pokorny, B. (2006). Roe deer-vehicle collisions in Slovenia: situation, mitigation strategy and countermeasures. Veterinarski Arhiv, 76, 177-187.

Quintero-Ángel, A., Osorio-Dominguez, D., VargasSalinas, F., \& Saavedra-Rodríguez, C. A. (2012). Roadkill rate of snakes in a disturbed landscape of Central Andes of Colombia. Herpetology Notes, 5, 99-105.

Ramírez, L. N. (2012). El parque automotor en la República Bolivariana de Venezuela 1990-2011, estratos medios de la población y elecciones 2012. Mundo Universitario, 10, 38-48.

Ramo, C., \& Busto, B. (1986). Influencia de las carreteras sobre la mortalidad de la fauna silvestre en el área Guanare-Masparro. Revista Unellez de Ciencia y Tecnología, 4, 33-38.

Rodda, G. (1989). Highway madness revisited: Roadkilled Iguana iguana in the llanos of Venezuela. Journal of Herpetology, 24(2), 201-211.

Rodríguez, J. P., \& Rojas-Suárez, F. (2008). Libro Rojo de la fauna Venezolana. Caracas: Provita y Shell de Venezuela. S.A. Caracas.

Rosa, A. O., \& Mauhs, J. (2004). Atropelamiento de animais silvestres na rodovia RS-040. Cuaderno de Pesquisa Série Biologia, 16(1), 35-42.

Santana, G. S. (2012). Factores influentes sobre atropelamientos de vertebrados na regiao central do estado do Rio Grande do Sul, Brasil. Neotropical Biology and Conservation, 7(1), 26-40. doi: 10.4013/ nbc.2012.71.05.

Santos, X., Llorente, G. A., Montori, A., Carretero, M. A., Franch, M., Garriga, N., \& Ritcher-Boix, A. (2007). Evaluating factors affecting amphibian mortality on roads: the case of the Common Toad Bufo bufo, near a breeding place. Animal Biodiversity and Conservation, 30(1), 97-104.

Solórzano, N., Escalona, M., Zambrano, C., Aranda, N., Molina, J. M., \& Blanco, L. (2006). Inventario de árboles en potreros en fincas del municipio Guanarito, estado Portuguesa. Revista Unellez de Ciencia y Tecnología, 24, 8-16.

Spellerberg, I. F. (1998). Ecological effects of roads and traffic: A literature review. Global Ecology and Biogeography, 7(5), 317-333.

Trombulak, S. C., \& Frissell, C. A. (2000). Review of ecological effects of roads on terrestrial and aquatic communities. Conservation Biology, 14(1), 18-30.

Turci, L. C. B., \& Bernarde, P. S. (2009). Vertebrados atropelados na rodovia estadual 383 em Rondônia, Brasil. Biotemas, 22(1), 121-127.

Vargas-Salinas, F., Delgado-Ospina, I., \& López-Aranda, F. (2011). Mortalidad por atropello vehicular y distribución de anfibios y reptiles en un bosque subandino en el occidente de Colombia. Caldasia, 33(1), 121-138. 


\section{APÉNDICE I}

Reptiles localizados atropellados en la Carretera Guanare-Guanarito (Portuguesa, Venezuela) durante presente estudio (columna A) y durante el estudio de Pacheco (1993) (columna B)

\section{APPENDIX I}

Reptiles found dead along the road Guanare-Guanarito (Portuguesa, Venezuela) during the present study (column A) and by Pacheco (1993) (column B)

\begin{tabular}{|c|c|c|c|c|}
\hline Orden & Familia & Especie & A & B \\
\hline CROCODILIA & Alligatoridae & Caiman crocodilus & 33 & 55 \\
\hline TESTUDINES & Podocnemidae & Podocnemis vogli & 5 & 6 \\
\hline \multirow[t]{25}{*}{ SQUAMATA } & Amphisbaenidae & Amphisbaena alba & - & 1 \\
\hline & Boidae & Boa constrictor & 5 & 16 \\
\hline & & Corallus hortulanus & - & 2 \\
\hline & & Epicrates cenchria & 6 & 21 \\
\hline & & Eunectes murinus & 2 & - \\
\hline & Colubridae & Chironius carinatus & 4 & 18 \\
\hline & & Clelia clelia & 3 & 3 \\
\hline & & Helicops angulatus & 25 & 1 \\
\hline & & Imantodes cenchoa & 1 & 1 \\
\hline & & Leptodeira annulata & 119 & 74 \\
\hline & & Leptophis ahaetulla & - & 2 \\
\hline & & Liophis lineatus & 10 & 7 \\
\hline & & Liophis melanotus & 3 & 2 \\
\hline & & Oxyrhopus petolarius & 3 & 4 \\
\hline & & Pseudoboa neuwiedii & 23 & 7 \\
\hline & & Sibon nebulata & 1 & 2 \\
\hline & & Spilotes pullatus & - & 3 \\
\hline & & Thamnodynastes sp. & - & 8 \\
\hline & Viperidae & Bothrops sp. & 9 & 4 \\
\hline & & Crotatus durissus & - & 3 \\
\hline & Serpientes no ident. & & 10 & 2 \\
\hline & Iguanidae & Iguana iguana & 4 & 14 \\
\hline & Teiidae & Ameiva ameiva & - & 8 \\
\hline & & Tupinambis teguixin & 1 & 15 \\
\hline & Lagartos no identificado & & 1 & - \\
\hline Total de individuos & & & 268 & 279 \\
\hline Total de especies* & & & 18 & 25 \\
\hline
\end{tabular}

* En el total de especies no se incluyen las no identificadas. 


\section{APÉNDICE II}

Aves atropelladas en la Carretera Guanare-Guanarito (Portuguesa, Venezuela) durante presente estudio (columna A) y el de Pacheco (1993) (columna B)

\section{APPENDIX II}

Birds found dead along the road Guanare-Guanarito (Portuguesa, Venezuela) during the present study (column A) and by Pacheco (1993) (column B)

\begin{tabular}{|c|c|c|c|c|}
\hline Orden & Familia & Especie & A & B \\
\hline SULIFORMES & Phalacrocoracidae & Phalacrocorax brasilianus & - & 1 \\
\hline \multirow[t]{7}{*}{ PELECANIFORMES } & \multirow[t]{4}{*}{ Ardeidae } & Ardea alba & - & 2 \\
\hline & & Bubulcus ibis & 1 & 4 \\
\hline & & Pilherodius pileatus & - & 1 \\
\hline & & Tigrisoma lineatum & - & 1 \\
\hline & \multirow[t]{3}{*}{ Theskiornithidae } & Mesembrinibis cayennensis & - & 1 \\
\hline & & Phimosus infuscatus & 1 & - \\
\hline & & Plegadis falcinellus & - & 1 \\
\hline \multirow[t]{5}{*}{ ACCIPITRIFORMES } & \multirow[t]{5}{*}{ Accipitridae } & Buteogallus meridionalis & - & 3 \\
\hline & & Buteogallus sp. & - & 1 \\
\hline & & Geranospiza caerulescens & - & 2 \\
\hline & & Rupornis magnirostris & 1 & 3 \\
\hline & & Accipitridae no ident. & - & 1 \\
\hline \multirow[t]{3}{*}{ CATHARTIFORMES } & \multirow[t]{3}{*}{ Cathartidae } & Cathartes aura & 8 & 3 \\
\hline & & Cathartes sp. & - & 1 \\
\hline & & Coragyps atratus & 9 & 16 \\
\hline \multirow{3}{*}{ FALCONIFORMES } & \multirow[t]{3}{*}{ Falconidae } & Caracara cheriway & 1 & 3 \\
\hline & & Falco sparverius & - & 1 \\
\hline & & Milvago chimachima & 2 & 1 \\
\hline \multirow[t]{2}{*}{ GRUIFORMES } & \multirow[t]{2}{*}{ Rallidae } & Aramides cajaneus & 1 & 5 \\
\hline & & Porphyrio martinicus & 1 & 3 \\
\hline CHARADRIIFORMES & Jacanidae & Jacana jacana & 2 & 1 \\
\hline \multirow[t]{4}{*}{ COLUMBIFORMES } & \multirow[t]{4}{*}{ Columbidae } & Columbina minuta & - & 7 \\
\hline & & Columbina squammata & - & 1 \\
\hline & & Columbina talpacoti & 3 & 25 \\
\hline & & Leptotila verreauxi & - & 1 \\
\hline \multirow[t]{2}{*}{ PSITTACIFORMES } & \multirow[t]{2}{*}{ Psittacidae } & Amazona ochrocephala & 1 & - \\
\hline & & Brotogeris jugularis & - & 1 \\
\hline \multirow[t]{3}{*}{ CUCULIFORMES } & \multirow[t]{3}{*}{ Cuculidae } & Crotophaga ani & 3 & 15 \\
\hline & & Crotophaga major & - & 1 \\
\hline & & Crotophaga sulcirostris & - & 2 \\
\hline CAPRIMULGIFORMES & Caprimulgidae & Nyctidromus albicollis & 4 & 1 \\
\hline \multirow[t]{6}{*}{ STRIGIFORMES } & \multirow[t]{5}{*}{ Strigidae } & Asio sp. & - & 1 \\
\hline & & Bubo virginianus & - & 1 \\
\hline & & Ciccaba virgata & - & 1 \\
\hline & & Pseudoscops clamator & 3 & 1 \\
\hline & & Strigidae no identificado & - & 1 \\
\hline & Tytonidae & Tyto alba & 8 & 10 \\
\hline APODIFORMES & Trochilidae & Phaethornis augusti & - & 1 \\
\hline CORACIIFORMES & Alcedinidae & Chloroceryle aenea & 1 & - \\
\hline \multirow[t]{2}{*}{ PASSERIFORMES } & Emberizidae & Ammodramus sp. & - & 1 \\
\hline & Furnaridae & Automolus rufipileatus & - & 1 \\
\hline
\end{tabular}


APÉNDICE II (CONTINUACIÓN) / APPENDIX II (CONTINUED)

\begin{tabular}{|c|c|c|c|c|}
\hline Orden & Familia & Especie & A & $\mathrm{B}$ \\
\hline & \multirow{7}{*}{ Icteridae } & Certhiaxis cinnamomeus & - & 1 \\
\hline & & Phacellodomus rufifrons & 2 & 1 \\
\hline & & Synallaxis albescens & - & 2 \\
\hline & & Synallaxis sp. & - & 1 \\
\hline & & Cacicus cela & 1 & - \\
\hline & & Icterus icterus & - & 1 \\
\hline & & Quiscalus lugubris & 1 & 5 \\
\hline & Mimidae & Mimus gilvus & 1 & - \\
\hline & Parulidae & Dendroica petechia & 1 & 1 \\
\hline & \multirow[t]{11}{*}{ Thraupidae } & Coereba flaveola & - & 1 \\
\hline & & Nemosia pileata & - & 1 \\
\hline & & Oryzoborus crassirostris & - & 1 \\
\hline & & Paroaria gularis & - & 3 \\
\hline & & Sicalis flaveola & 1 & 3 \\
\hline & & Sporophila minuta & - & 1 \\
\hline & & Sporophila plumbea & - & 2 \\
\hline & & Sporophila sp. & - & 1 \\
\hline & & Thachyphonus rufus & - & 1 \\
\hline & & Thraupis episcopus & - & 1 \\
\hline & & Volatinia jacarina & - & 2 \\
\hline & Donacobidae & Donacobius atricapilla & - & 3 \\
\hline & \multirow[t]{6}{*}{ Tyrannidae } & Elaenia flavogaster & - & 2 \\
\hline & & Myiozetetes cayenensis & 2 & - \\
\hline & & Myiozetetes similis & - & 1 \\
\hline & & Pitangus sulphuratus & 1 & 4 \\
\hline & & Todirostrum cinereum & - & 4 \\
\hline & & Tyrannus melancholicus & - & 1 \\
\hline & \multicolumn{2}{|l|}{ Aves no identificadas } & 6 & - \\
\hline Total de individuos & & & 66 & 171 \\
\hline Total de especies* & & & 25 & 61 \\
\hline
\end{tabular}

* En el total de especies no se incluyen las no identificadas. 


\section{APÉNDICE III}

Mamíferos localizadas atropellados en la Carretera Guanare-Guanarito (Portuguesa, Venezuela) durante presente estudio (columna A) y durante el estudio de Pacheco (1993) (columna B)

APPENDIX III

Mammals found dead along the road Guanare-Guanarito (Portuguesa, Venezuela) during the present study (column A) and by Pacheco (1993) (column B)

\begin{tabular}{|c|c|c|c|c|}
\hline Orden & Familia & & A & B \\
\hline DIDELPHIMORPHIA & Didelphidae & Didelphis marsupialis & 39 & 74 \\
\hline CINGULATA & Dasypodidae & Dasypus novemcinctus & 13 & 8 \\
\hline \multirow[t]{2}{*}{ PILOSA } & Myrmecophagidae & Myrmecophaga tridactyla & 3 & 1 \\
\hline & & Tamandua tetradactyla & 13 & 24 \\
\hline PRIMATES & Atelidae & Alouatta seniculus & - & 3 \\
\hline LAGOMORPHA & Leporidae & Sylvilagus floridanus & 11 & 5 \\
\hline \multirow[t]{8}{*}{ RODENTIA } & Caviidae & Hydrochoerus hydrochaeris & 1 & 1 \\
\hline & Cuniculidae & Cuniculus paca & - & 1 \\
\hline & Dasyproctidae & Dasyprocta leporina & - & 3 \\
\hline & Erethizontidae & Coendou perhensilis & - & 1 \\
\hline & Cricetidae & Sigmodon alstoni & - & 1 \\
\hline & & Zygodontomys sp. & - & 1 \\
\hline & & Cricetidae no identificado & - & 3 \\
\hline & Sciuridae & Sciurus granatensis & 1 & - \\
\hline \multirow[t]{3}{*}{ CHIROPTERA } & Molossidae & Molossidae no identificado & - & 1 \\
\hline & Noctilionidae & Noctilio leporinus & 1 & 3 \\
\hline & Phyllostomidae & Phyllostomus hastatus & 1 & - \\
\hline \multirow[t]{9}{*}{ CARNIVORA } & Canidae & Canis familiaris & 15 & - \\
\hline & & Cerdocyon thous & 9 & 61 \\
\hline & Felidae & Felis catus & 10 & - \\
\hline & & Leopardus pardalis & - & 1 \\
\hline & & Puma yagouaroundi & 1 & 2 \\
\hline & Mephitidae & Conepatus semistriatus & 1 & 2 \\
\hline & Mustelidae & Eira barbara & - & 1 \\
\hline & & Galictis vittata & - & 5 \\
\hline & Procyonidae & Procyon cancrivorus & 9 & 8 \\
\hline Mamíf. no ident. (2 ) & & & 2 & - \\
\hline Total de individuos & & & 130 & 210 \\
\hline Total de especies* & & & 15 & 21 \\
\hline
\end{tabular}

* En el total de especies no se incluyen las no identificadas. 